\title{
POLISH CREDIT INSTITUTIONS WITHIN THE EUROPEAN UNION: A CROSS-COUNTRY SURVEY
}

\author{
MARIUSZ DYBAE*
}

This article aims to analyze the importance and evolution of credit institutions, not only within the Polish financial system, but throughout the European Union.

The financial system consists of many different financial institutions. Information on changes in the number of financial institutions in the period 1992-2009 is presented in the first table (Table 1). Table 1 demonstrates that in terms of quantity cooperative banks enjoy the dominant position, although their number decreased over the period, from 1,653 to 576. The number of commercial banks only declined from 87 to 67 . The drop in the number for pension funds (from 21 to 14) is observed too. Insurance companies and credit unions evolved in a similar manner. First, a noticeable rapid growth, from 25 to 78 then a drop to 65 (insurance companies), and from 32 to 228 then a decline to 62 (credit unions). Investment funds and their management companies were the only institutions to see steady growth. Their number increased from 1 to 369 in the case of funds, and from 1 to 43 in the case of management companies. The number of brokerage houses in the period 1996-2009 increased from 50 to 59.

Share of the financial system in terms of number of institutions does not translate into share in terms of assets (Table 2). While the value of assets in the period 1996-2009 increased more than sevenfold, the increase was not equal. The dominant position was held by commercial banks, although their share did decrease, from $94 \%$ to $71 \%$. The share of cooperative banks and broker entities also declined, from $4.4 \%$ and $0.9 \%$ to $4.1 \%$ and $0.7 \%$, respectively. On the other hand, insurance companies, investment funds and credit unions recorded an increase in share of the financial system in terms of assets, from $3.9 \%, 0.7 \%$ and $0.1 \%$ to $9.3 \%, 6.3 \%$ and $0.8 \%$, respectively. However, the most dynamic position was occupied by the pension funds, which increased their share of the financial system from $0 \%$ to $12 \%$.

The structure of the Polish financial system is no different to that of the European Union (EU) ${ }^{1}$ in that the main element of the financial system of the enlarged European Union (EU27) is credit institutions ${ }^{2}$ too. At the end of 2009 credit institutions held assets of more

DOI: $10.2478 /$ wrlae-2013-0042

* PhD; LLM; Institute of Economics, Department of Finance Management, University of Wroclaw. mariusz_dybal@prawo.uni.wroc.pl

${ }^{1}$ Unless otherwise stated, the European Union, or EU, or EU27, consists of 27 countries, as at 1 January 2011.

${ }^{2}$ The number of credit institutions in each Member State includes the credit institutions under the law of that country, regardless or whether or not they are subsidiaries of foreign banks, and the branches of foreign banks in 
than EUR 42 tn. This means an increase in assets compared to 2001 of more than EUR 17 bn (an increase of $70.9 \%$ ).

The data show (Table 3) a change over the years in the country holding the position of undisputed leader in terms of share of its banking system in the banking system across the European Union. In terms of the value of the assets of credit institutions, Germany was overtaken by the United Kingdom. Both countries recorded a decline in the share of their assets in the assets of the EU, with Germany's share falling from $25.4 \%$ to $17.6 \%$ and the share of the United Kingdom from $23.6 \%$ to $22.35 \%$.

Polish banking sector assets increased in the period 2001-9 from EUR 133 bn to EUR 274 bn (an increase of 105.4\%). Taking into account the share of the Polish banking sector in the EU, this meant an increase from $0.5 \%$ in 2001 to $0.7 \%$ in 2009 . These data are not impressive compared to the so-called 'old' ${ }^{3}$ EU countries. However, it should be noted that when considering only the "new' ${ }^{4}$ member states this was the highest score. In none of the remaining 11 new EU countries were the assets of the banking system higher.

Another parameter which can be used to evaluate the banking sector across the countries is the share of its assets in Gross Domestic Product (GDP). For this aspect in Poland in the period 2001-9, an increase was recorded, from $63 \%$ to $88 \%$ (Table 3). But this was far below the EU average - $261 \%$ in 2001 and $357 \%$ in 2009 - not to mention the outcome for Luxembourg (at the beginning and end of the period respectively, 3,194\% and 2,118\%). In fact, in recent years Poland was clearly in one of the last places within both the EU15 and the NMS. In the period 2001-9, Poland came third from last of the entire European Union in terms of banking sector assets compared to GDP, in 2001 just ahead of Lithuania (32\% of GDP) and probably Romania. In 2009 Poland was ahead of Slovakia (86\% of GDP) and Romania (75\% of GDP). Among the 12 New Member States the highest rate of banking sector assets to GDP was reached every time in Cyprus. In 2001 this was at the rate of 399\%, while in 2009 it rose to $822 \%$.

Among the largest 25 banks in Europe in terms of assets no bank from Poland will be found (Table 4). The French BNP Paribas was the largest bank in 2009, its assets exceeding USD 2,952 bn. BNP Paribas overtook another two banks from the United Kingdom, Royal Bank of Scotland and HSBC Holdings respectively. The smallest on the list turned out to be the Italian Banca MPS, which slightly exceeded in assets USD 292 bn. In the top ten were as many as four banks from the United Kingdom and three French banks. The group was rounded out by Germany, the Netherlands and Italy, with a single bank from each country.

Compared to the 25 biggest banks in Europe, the largest Polish in terms of assets, PKO Bank Polski, is very small (BNP Paribas holds assets more than 50 times greater than PKO Bank Polski). Nevertheless, this is the largest bank (assets of EUR $37.4 \mathrm{bn}$ ) of the 25 largest in Central and East Europe - the New Member States. In second place in 2009 was another Polish bank, Bank Pekao SA, with assets of EUR 30.895 bn, and third was Ceskoslovenska obchodní banka, with EUR 28.444 bn. The smallest in the group of 25 banks on the list was Slovakia Tatra banka, which slightly exceeded in assets EUR 9 bn.

\footnotetext{
that Member State. Unless otherwise stated, the credit institutions in Poland are the domestic banks, both commercial and cooperative.

${ }^{3}$ The 'Old', or EU15, means the European Union consisting of Belgium, Denmark, Germany, Ireland, Greece, Spain, France, Italy, Luxembourg, the Netherlands, Austria, Portugal, Finland, Sweden and the United Kingdom. ${ }^{4}$ The 'New', or NMS (New Member States), consists of Bulgaria, the Czech Republic, Estonia, Cyprus, Latvia, Lithuania, Hungary, Malta, Poland, Romania, Slovenia and Slovakia.
} 
In the top ten were as many as four banks from Poland (PKO Bank Polski, Bank Pekao SA, BRE Bank and ING Bank) and three from the Czech Republic. The group was rounded out by Hungary, Slovenia and Romania, with a single bank from each country.

Overall, in the list of the 25 largest banks in Central and East Europe there were seven from Poland (other than those already mentioned, BZ WBK, Bank Millennium and Kredyt Bank). Six banks came from Hungary, four from the Czech Republic, three from Slovakia and two from Romania. The group was rounded out by Hungary, Slovenia and Romania, each country with a single bank.

The assets of Polish banks in total amounted to EUR 133.3 bn in 2009. This meant that considered against all 25 banks from Central and East Europe, Polish banks have reached the largest regional share in the assets, of $35 \%$. But even a hypothetical bank of this size would not be seen on the list of the 25 largest banks in the European Union. If we aggregate the assets of the 25 Central and Eastern European banks, we would obtain a bank with assets amounting to EUR 380.5 bn. This would take 19th place among the 25 largest banks in the EU, with the resulting bank holding only $19.3 \%$ of the largest bank in the EU in terms of assets.

The scale of the market concentration of credit institutions in the EU was presented with the share of the five banks with the greatest assets in total assets of credit institutions (Table 5). This was designed such that the indicator is called the CR5.

In terms of the CR5 Poland in 2001 amounted to $54.7 \%$, and $43.9 \%$ in 2009. This means movement from the group of countries with a CR5 rate above the weighted average for the European Union to a group of less than the weighted average for the European Union. Considering only the New Member States, the Polish credit institution sector was marked every time by the smallest degree of concentration. The most concentrated market was in Estonia, where five credit institutions controlled $98.9 \%$ of the total market in 2001 and $93.4 \%$ in 2009. At the other extreme was the banking sector in Germany, where the CR5 rate stood at $20.2 \%$ in 2001 and $25 \%$ in 2009 .

The next section provides information on the share of foreign credit institutions in total assets (Table 6). The average for the European Union in the years 2001-9 increased from $23 \%$ to $26 \%$. The smallest market share of foreign credit institutions came in the Netherlands, at $2 \%$ and 5\% respectively in 2001 and 2009. In turn, Slovakia and Estonia are countries in which foreign banks have the largest share. In 2001 80\% (Slovakia) and 91\% (Estonia), and in 2009 96\% (Slovakia) and 95\% (Estonia).

Poland was characterized during the period by stability in data. In 2001 banks with foreign capital held $69 \%$ of the market measured in assets. At the end of 2009 share fell to $68 \%$. The highest share was in 2008 (72\%), the lowest in 2004-5 (67\%). Generally, the countries of the EU15 were characterized by a lower value for the rate of foreign credit institutions in assets, compared to the countries of the New Member States.

The following table (Table 7) shows the number of credit institutions operating in different countries. The number of credit institutions operating over the years fell from 9,747 in 2001 to 8,358 in 2009. In the period 2001-9 the highest number of credit institutions was observed in Germany (2,526 institutions in 2001 and 1,948 in 2009), the smallest in Estonia (seven credit institutions in 2001 and 18 in 2009).

Poland was found to be the fifth market in terms of number of credit institutions in the European Union. In 2001756 different lending institutions were recorded. However, in 2009 
there were only 710 credit institutions in Poland. The undeniable leader was Germany and then, in order, Italy, Austria and France.

The next table (Table 8) presents the number of branches of credit institutions in the European Union. In contrast to the number of institutions, number of establishments increased (from 206,724 in 2001 to 229,532 in 2009). In 2001 most units were recorded in Germany, 53,931, while in 2009 Spain was the leader with 44,431 units. In every year the lowest number of facilities was observed in Malta, 102 in 2001 and 116 in 2009.

Poland in 2001 achieved eighth place, with 4,080 outlets, but in 2009 was fifth, with 13,292 establishments. However, it should be noted that in the period 2005-9 the total number of credit institutions in Poland consisted of banks as well as credit unions.

The data on the number of inhabitants served by each bank branch in the European Union are presented in the next table (Table 9). In the period 2001-9, the data for the entire European Union were as follows: in 2001 there were 2,196 residents per local unit, in 2009 2,180; in 2001 the largest population per bank branch was in Lithuania $(22,314)$, the lowest in Cyprus (696), while in 2009 the largest population per bank branch was counted in Estonia $(6,291)$, the lowest again in Cyprus (858).

Poland in 2001, reaching 9,375 persons per bank branch, was second of the countries in terms of lack of facilities. In 2009 banking sector in Poland achieved the result of 2,867 people per bank branch, but after deducting Credit Unions this result would be closer to Estonia. Thus there is still potential for growth.

In the years 2001-2009 the credit institutions of the European Union employed annually some three million workers (Table 10). Although the number of employees fell from 772,100 in 2001 to 685,500 workers in 2008 , Germany remained the largest banking sector employer in the EU. In contrast, the smallest employer was Malta, where in 2001 there were only 3,584 employees of credit institutions, and 3,834 in 2009.

In Poland in 2001 168,529 workers were employed, this representing sixth place in the European Union. In 2009 Poland was also the sixth largest employer, with 183,064 workers in the banking sector. It was preceded, as at the beginning of the decade, by Germany, the United Kingdom, France, Italy and Spain.

However, the number of employees in the credit institution sector is not proportional to the assets held by the credit institutions. In the European Union in the years 2001-9 there was an increase of the assets per employee indicator (Table 11). In 2001 this was EUR 7,754,000 per employee, while in 2009 already EUR 20,995,000. In 2001 the highest assets per employee was counted for credit institutions in Luxembourg (EUR 30,175,000), the lowest in Lithuania (EUR 496,000). At the end of the period the highest assets per employee was recorded in Ireland (EUR 34,669,000), the lowest in Bulgaria (EUR 1,107,000).

In Poland in 2001 this was EUR 792,000 per employee, the second lowest outcome, although had Bulgaria and Romania been taken into account, it would probably would be fourth lowest. In 2009 the ratio increased to EUR 1,492,000 per employee. This allowed the third lowest place to be achieved (ahead of Romania and Bulgaria).

Data presented so far have indicated a growing share of the Polish banking sector considered against European credit institutions. One reason for this situation seems to be an increase in household financial assets.

The next table (Table 12) shows the financial assets of households in the years 2000-9. In 2000 assets were valued at PLN 280.4 bn. After nine years, assets had tripled, reaching PLN $859.2 \mathrm{bn}$. Investment in open pension funds (OFE) accounted for an important part of 
household financial assets. Mandatory contributions to pension funds meant that the assets of these institutions increased from PLN 2.3 bn to PLN 178.6 bn in the period 2001-9.

Financial assets of households increased not only in absolute terms, but also relative to GDP (Table 13). In 2000 the assets to GDP ratio stood at $37.7 \%$. In subsequent years there was a yearly increase (except for 2004 and 2008). In 2009 household financial assets relative to GDP was already at $63.9 \%$.

Financial assets of households replenished financial institutions in an uneven manner. The next table (Table 14) shows the structure of household financial assets in the years 20019. The data presents the decreasing role of bank deposits and treasury securities in favor of alternative financial instruments. Therefore share of treasury securities declined only from $4.8 \%$ to $1.5 \%$, while a decline in the share of bank deposits was seen in the change from $65.4 \%$ to $43.8 \%$. In addition, the proportion of cash in circulation decreased slightly (from $11.4 \%$ to $10.4 \%$ ).

Other elements of the structure of assets increased their shares: the share of funds deposited in financial instruments such as pension funds (from $5.8 \%$ to $20.8 \%$ ), investment funds (from $3 \%$ to $9.1 \%$ ), unit-linked assets and life insurance premiums (from $6.2 \%$ to $7.9 \%$ ), stock listed on the Warsaw Stock Exchange (from $2.9 \%$ to $4.9 \%$ ) and credit unions (from $0.5 \%$ to $1.3 \%$ ).

Development of the Polish financial system was made possible also thanks to the increasing wealth of Poles. The data on this subject are presented in the final table (Table 15), which shows GDP per capita in Purchasing Power Standards (PPS) in the years 2001-9 for all countries of the European Union. In 2001 the highest rate was achieved in Luxembourg (234\%), the lowest in Romania (28\%). In Poland GDP per capita in PPS amounted to $48 \%$ of average GDP per capita for the European Union. This result placed Poland 22nd out of 27 countries in 2001 .

In 2009 highest GDP per capita was again achieved in Luxembourg (271\%), the lowest this time in Bulgaria (44\%). In Poland GDP per capita in PPS amounted to $61 \%$ of average GDP per capita for the European Union. Despite a 13\% increase, the outcome placed Poland 23rd of the 27 members of the EU. Although there was an increase in the indicator, Poland was overtaken by other countries where growth was higher. It should, however, be noted that besides Bulgaria, the Czech Republic and Slovakia, Poland was the only country in which GDP per capita in PPS grew annually. 
Table 1 Number of financial institutions in Poland, 1992-2009

\begin{tabular}{|c|c|c|c|c|c|c|c|c|c|c|c|c|c|c|c|c|c|c|}
\hline & 1992 & 1993 & 1994 & 1995 & 1996 & 1997 & 1998 & 1999 & 2000 & 2001 & 2002 & 2003 & 2004 & 2005 & 2006 & 2007 & 2008 & 2009 \\
\hline $\begin{array}{l}\text { Commercial and cooperative } \\
\text { banks }\end{array}$ & n.a. & $\begin{array}{r}1 \\
740\end{array}$ & $\begin{array}{r}1 \\
694\end{array}$ & $\begin{array}{r}1 \\
591\end{array}$ & $\begin{array}{r}1 \\
475\end{array}$ & $\begin{array}{r}1 \\
378\end{array}$ & $\begin{array}{r}1 \\
272\end{array}$ & 858 & 754 & 713 & 667 & 660 & 653 & 649 & 647 & 645 & 649 & 643 \\
\hline Cooperative banks & n.a. & $\begin{array}{r}1 \\
653\end{array}$ & $\begin{array}{r}1 \\
612\end{array}$ & $\begin{array}{r}1 \\
510\end{array}$ & $\begin{array}{r}1 \\
394 \\
\end{array}$ & $\begin{array}{r}1 \\
295\end{array}$ & $\begin{array}{r}1 \\
189\end{array}$ & 781 & 680 & 642 & 605 & 600 & 596 & 588 & 584 & 581 & 579 & 576 \\
\hline Credit unions & n.a. & 32 & 106 & 137 & 168 & 198 & 220 & 228 & 146 & 144 & 120 & 109 & 83 & 75 & 70 & 67 & 62 & 62 \\
\hline Insurance companies & n.a. & 25 & 29 & 39 & 41 & 50 & 54 & 58 & 66 & 72 & 74 & 78 & 69 & 68 & 65 & 67 & 66 & 65 \\
\hline Investment funds & 1 & 1 & 1 & 4 & 5 & 20 & 38 & 62 & 81 & 94 & 124 & 137 & 154 & 190 & 241 & 277 & 319 & 369 \\
\hline $\begin{array}{l}\text { Investment fund management } \\
\text { companies }\end{array}$ & 1 & 1 & 1 & 2 & 5 & 10 & 14 & 15 & 21 & 17 & 19 & 16 & 20 & 23 & 26 & 33 & 39 & 43 \\
\hline Open pension funds & 0 & 0 & 0 & 0 & 0 & 0 & 0 & 21 & 21 & 17 & 16 & 16 & 15 & 15 & 15 & 15 & 14 & 14 \\
\hline Brokerage entities & n.a. & n.a. & n.a. & n.a. & 50 & 47 & 46 & 48 & 49 & 42 & 38 & 36 & 40 & 42 & 47 & 53 & 58 & 59 \\
\hline
\end{tabular}

Note: (n.a.) - not available

Source: Jacek Osiński, Paweł Wyczański, Dobiesław Tymoczko, Agnieszka Grąt (eds.), Rozwój systemu finansowego w Polsce w latach 2002-2003 (NBP 2004) 19; Jacek Osiński, Dobiesław Tymoczko (eds.), Rozwój systemu finansowego w Polsce w 2005r. (NBP 2005) 14; Paweł Sobolewski, Dobiesław Tymoczko, Rozwój systemu finansowego $w$ Polsce $w$ 2009r. (NBP 2010) 9;

$<$ http://www.skok.pl/?4DgU5kDcljUBKlujjqqMbq4hwn9YYxQE3m3uRpgHm7U=;VV50994D95FE> accessed 15 March 2011; < http:// www.knf.gov.pl> accessed 10 March 2011; 'Stowarzyszenie Towarzystw Funduszy Inwestycyjnych. Raport 1999' (STFI 2000) 6; 'Stowarzyszenie Towarzystw Funduszy Inwestycyjnych. Raport 2002’ (STFI 2002) 14

Table 2 Assets of financial institutions in Poland, 1996-2009 (PLN bn)

\begin{tabular}{|l|r|r|r|r|r|r|r|r|r|r|r|r|r|r|}
\hline & $\mathbf{1 9 9 6}$ & $\mathbf{1 9 9 7}$ & $\mathbf{1 9 9 8}$ & $\mathbf{1 9 9 9}$ & $\mathbf{2 0 0 0}$ & $\mathbf{2 0 0 1}$ & $\mathbf{2 0 0 2}$ & $\mathbf{2 0 0 3}$ & $\mathbf{2 0 0 4}$ & $\mathbf{2 0 0 5}$ & $\mathbf{2 0 0 6}$ & $\mathbf{2 0 0 7}$ & $\mathbf{2 0 0 8}$ & $\mathbf{2 0 0 9}$ \\
\hline Commercial banks & 197.1 & 247.7 & 318.7 & 363.4 & 428.4 & 469.7 & 466.5 & 489.3 & 538 & 587 & 681.8 & 792.8 & 1039.1 & 1059.6 \\
\hline Cooperative banks & 9.1 & 11.3 & 13.7 & 15.4 & 18 & 21.5 & 23.4 & 25.7 & 28.7 & 33.9 & 42.1 & 48.9 & 56.5 & 61.7 \\
\hline Credit unions & 0.2 & 0.4 & 0.6 & 0.9 & 1.2 & 1.8 & 2.5 & 3.4 & 4.2 & 5.3 & 6.0 & 7.3 & 9.4 & 11.6 \\
\hline Insurance companies & 8.1 & 13.2 & 20.7 & 28.9 & 37.9 & 48 & 57.5 & 65.7 & 77.5 & 89.6 & 108.6 & 126.9 & 137.9 & 139 \\
\hline Investment funds & 1.4 & 1.9 & 1.8 & 3.1 & 9.5 & 12.1 & 22.8 & 33.2 & 37.7 & 61.6 & 99.2 & 133.8 & 73.9 & 93.4 \\
\hline Open pension funds & 0 & 0 & 0 & 2.3 & 9.9 & 19.4 & 31.6 & 44.8 & 62.6 & 86.1 & 116.6 & 140 & 138.3 & 178.6 \\
\hline Brokerage entities & 1.8 & 3 & 3.2 & 3.6 & 3.9 & 2.9 & 2.8 & 3.7 & 5.5 & 6.9 & 10.8 & 11.8 & 8.6 & 9.9 \\
\hline \multicolumn{2}{r}{} & & & & & &
\end{tabular}

Source: Jacek Osiński, Paweł Wyczański, Dobiesław Tymoczko, Agnieszka Grąt (eds.), Rozwój systemu finansowego w Polsce w latach 2002-2003 (NBP 2004) 19; Jacek Osiński, Dobiesław Tymoczko (eds.), Rozwój systemu finansowego w Polsce w 2005r. (NBP 2006) 14; Paweł Sobolewski, Dobiesław Tymoczko, Rozwój systemu finansowego w Polsce w 2009r. (NBP 2010) 9;

<http://www.skok.pl/?4DgU5kDcljUBKlujjqqMbq4hwn9YYxQE3m3uRpgHm7U=;VV50994D95FE > accessed 15 March 2011; < http:// www.knf.gov.pl> accessed 10 March 2011; 'Stowarzyszenie Towarzystw Funduszy Inwestycyjnych. Raport 1999' (STFI 2000) 6; 'Stowarzyszenie Towarzystw Funduszy Inwestycyjnych. Raport 2002’ (STFI 2002) 14 


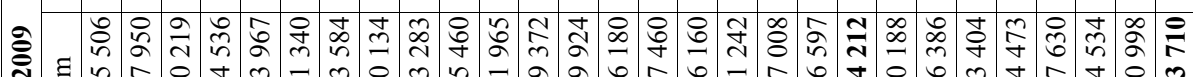

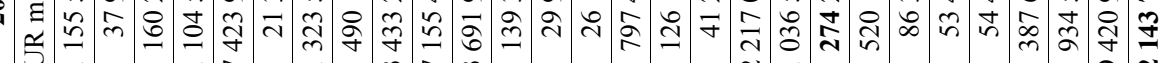

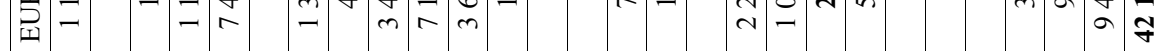

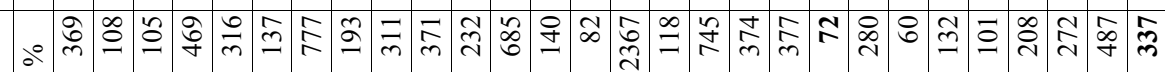

帘

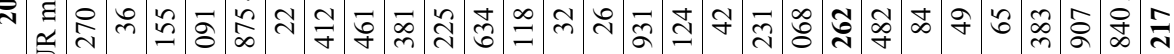
恿

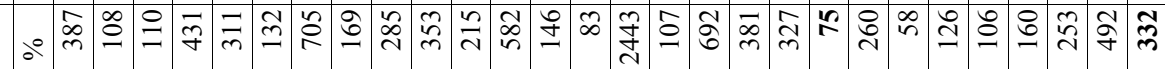

帘

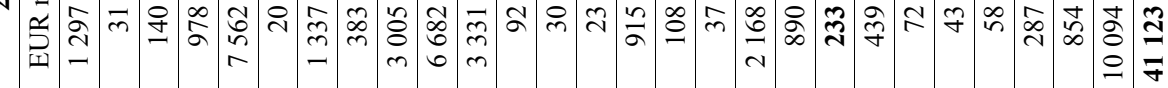

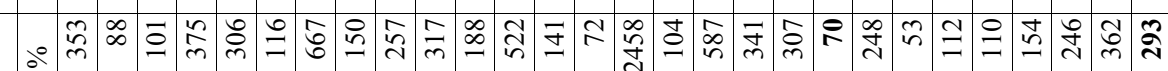
‡

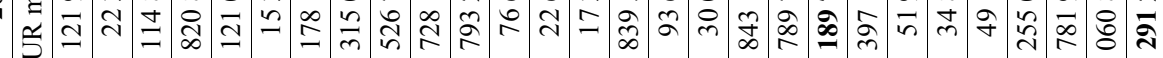

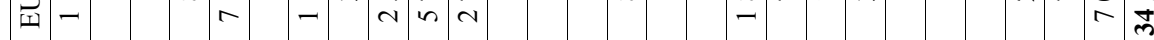

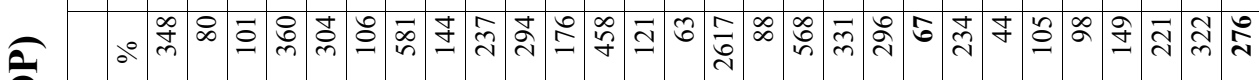
(1)

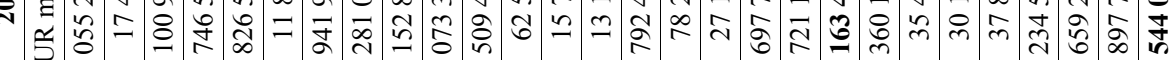

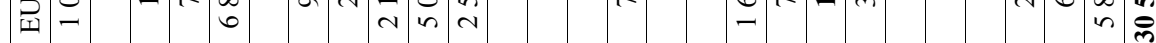

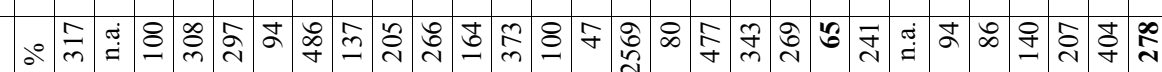

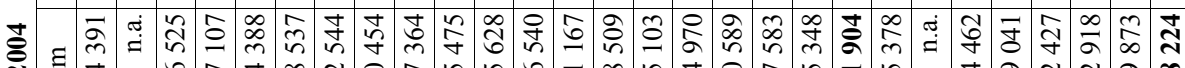

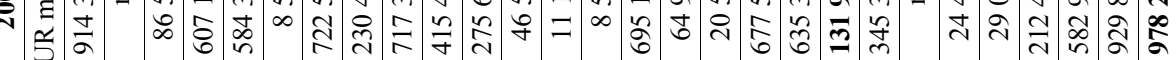

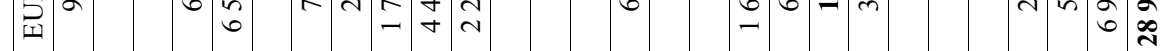

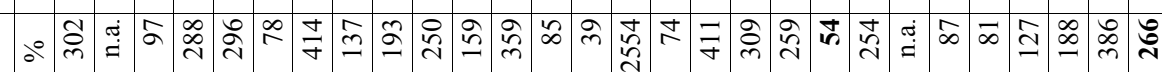

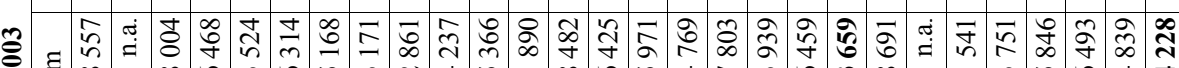

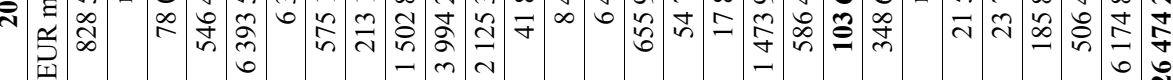

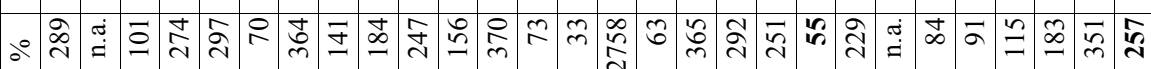

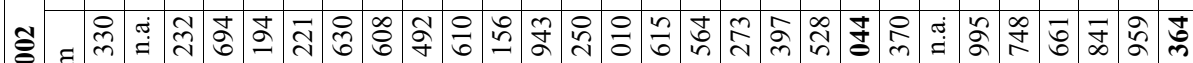

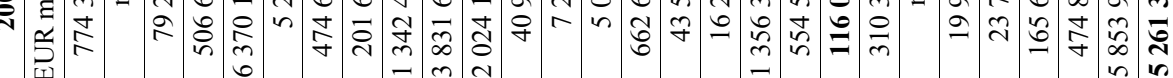
০

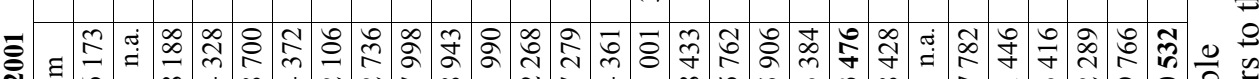

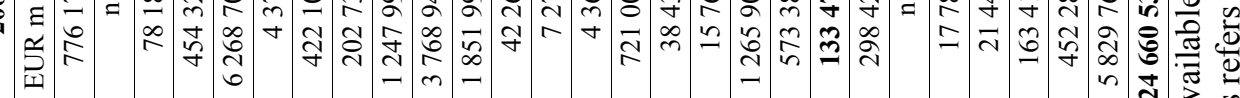




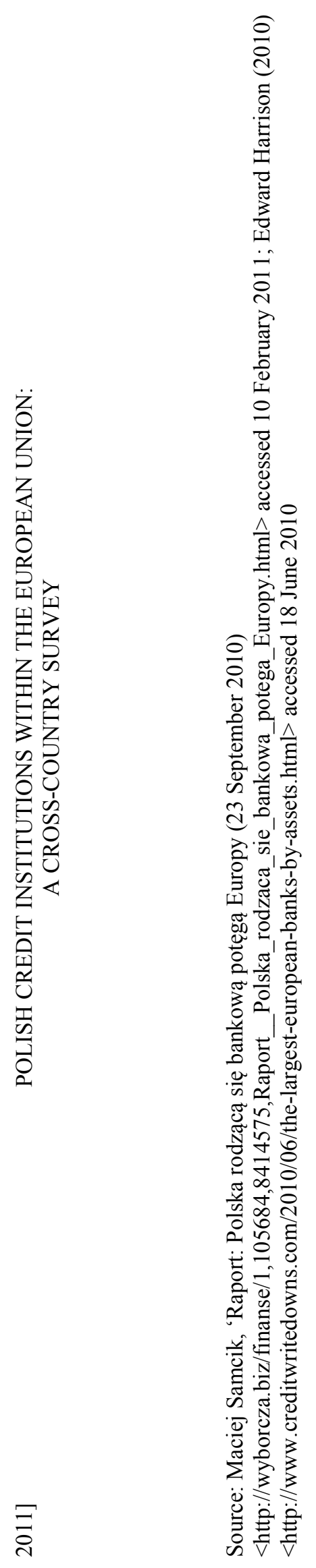


Table 5 The share of the five largest credit institutions in total assets (CR5), in \%

\begin{tabular}{|c|c|c|c|c|c|c|c|c|c|}
\hline & 2001 & 2002 & 2003 & 2004 & 2005 & 2006 & 2007 & 2008 & 2009 \\
\hline Belgium & 78.3 & 82.0 & 83.5 & 84.3 & 85.3 & 84.4 & 83.4 & 80.8 & 77.1 \\
\hline Bulgaria & n.a. & n.a. & n.a. & n.a. & 50.8 & 50.3 & 56.7 & 57.3 & 58.3 \\
\hline Czech Republic & 68.4 & 65.7 & 65.8 & 64.0 & 65.5 & 64.1 & 65.7 & 62.1 & 62.4 \\
\hline Denmark & 67.6 & 68.0 & 66.6 & 67.0 & 66.3 & 64.7 & 64.2 & 66.0 & 64.0 \\
\hline Germany & 20.2 & 20.5 & 21.6 & 22.1 & 21.6 & 22.0 & 22.0 & 22.7 & 25.0 \\
\hline Estonia & 98.9 & 99.1 & 99.2 & 98.6 & 98.1 & 97.1 & 95.7 & 94.8 & 93.4 \\
\hline Ireland & 42.5 & 46.1 & 44.4 & 43.9 & 47.8 & 49.0 & 50.4 & 55.3 & 58.8 \\
\hline Greece & 67.0 & 67.4 & 66.9 & 65.0 & 65.6 & 66.3 & 67.7 & 69.5 & 69.2 \\
\hline Spain & 43.9 & 43.5 & 43.1 & 41.9 & 42.0 & 40.4 & 41.0 & 42.4 & 43.3 \\
\hline France & 47.0 & 44.6 & 46.7 & 49.2 & 51.9 & 52.3 & 51.8 & 51.2 & 47.2 \\
\hline Italy & 29.0 & 30.5 & 27.5 & 26.4 & 26.8 & 26.2 & 33.1 & 33.0 & 34.0 \\
\hline Cyprus & 61.3 & 57.8 & 57.2 & 57.3 & 59.8 & 63.9 & 64.9 & 63.8 & 65.0 \\
\hline Latvia & 63.4 & 65.3 & 63.1 & 62.4 & 67.3 & 69.2 & 67.2 & 70.2 & 69.3 \\
\hline Lithuania & 87.6 & 83.9 & 81.0 & 78.9 & 80.6 & 82.5 & 80.9 & 81.3 & 80.5 \\
\hline Luxembourg & 28.0 & 30.3 & 31.8 & 29.7 & 30.7 & 29.1 & 27.9 & 27.3 & 27.8 \\
\hline Hungary & 56.4 & 54.5 & 52.1 & 52.7 & 53.2 & 53.5 & 54.1 & 54.4 & 55.2 \\
\hline Malta & 81.1 & 82.4 & 77.7 & 78.5 & 75.3 & 70.9 & 70.2 & 72.8 & 72.7 \\
\hline Netherlands & 82.5 & 82.7 & 84.2 & 84.0 & 84.5 & 85.1 & 86.3 & 86.8 & 85.0 \\
\hline Austria & 44.9 & 45.6 & 44.2 & 43.8 & 45.0 & 43.8 & 42.8 & 39.0 & 37.2 \\
\hline Poland & 54.7 & 53.4 & 52.3 & 50.2 & 48.5 & 46.1 & 46.6 & 44.2 & 43.9 \\
\hline Portugal & 59.8 & 60.5 & 62.7 & 66.5 & 68.8 & 67.9 & 67.8 & 69.1 & 70.1 \\
\hline Romania & n.a. & n.a. & n.a. & n.a. & 59.4 & 60.1 & 56.3 & 54.0 & 52.4 \\
\hline Slovenia & 67.6 & 68.4 & 66.4 & 64.6 & 63.0 & 62.0 & 59.5 & 59.1 & 59.7 \\
\hline Slovakia & 66.1 & 66.4 & 67.5 & 66.5 & 67.7 & 66.9 & 68.2 & 71.6 & 72.1 \\
\hline Finland & 79.5 & 78.6 & 81.2 & 82.7 & 82.9 & 82.3 & 81.2 & 82.8 & 82.6 \\
\hline Sweden & 54.6 & 56.0 & 53.8 & 54.4 & 57.3 & 57.8 & 61.0 & 61.9 & 60.7 \\
\hline United Kingdom & 28.6 & 29.6 & 32.8 & 34.5 & 36.3 & 35.9 & 40.7 & 36.5 & 40.8 \\
\hline EU27* weighted average & 37.8 & 38.3 & 39.7 & 40.9 & 42.6 & 41.5 & 41.5 & 45.2 & 44.3 \\
\hline EU27* unweighted average & 59.1 & 59.3 & 58.9 & 58.8 & 59.3 & 59.0 & 59.5 & 59.6 & 59.5 \\
\hline
\end{tabular}

Note: (n.a.) - not available

Aggregate concentration figures display both weighted and unweighted averages

(*) For 2001-4 this refers to the EU15.

Source: European Central Bank, 'EU Banking Structures. September 2005' (European Central Bank 2005) 54;

European Central Bank, 'EU Banking Structures. September 2010’ (European Central Bank 2010) 36

Table 6 Asset share of credit institutions with majority foreign equity ownership (in \%)

\begin{tabular}{|c|c|c|c|c|c|c|c|c|c|}
\hline & 2001 & 2002 & 2003 & 2004 & 2005 & 2006 & 2007 & 2008 & 2009 \\
\hline Belgium & 25 & 24 & 24 & 23 & 23 & 25 & 25 & 27 & 61 \\
\hline Bulgaria & n.a. & n.a. & n.a. & n.a. & 76 & 77 & 82 & 83 & 84 \\
\hline Czech Republic & 77 & 93 & 96 & 96 & 97 & 97 & 91 & 91 & 90 \\
\hline Denmark & 23 & 19 & 23 & 20 & 20 & 20 & 19 & 18 & 20 \\
\hline Germany & 5 & 3 & 6 & 6 & 11 & 11 & 11 & 12 & 11 \\
\hline Estonia & 91 & 90 & 89 & 98 & 99 & 98 & 98 & 97 & 95 \\
\hline Ireland & 60 & 49 & 46 & 45 & 43 & 43 & 59 & 56 & 50 \\
\hline Greece & 9 & 21 & 10 & 27 & 28 & 37 & 23 & 22 & 21 \\
\hline Spain & 9 & 10 & 7 & 8 & 11 & 8 & 8 & 11 & 10 \\
\hline France & 13 & 13 & 11 & 11 & 12 & 11 & 12 & 12 & 11 \\
\hline Italy & 5 & 6 & 6 & 6 & 9 & 14 & 17 & 15 & 13 \\
\hline Cyprus & 20 & 20 & 21 & 25 & 27 & 29 & 32 & 38 & 37 \\
\hline Latvia & 34 & 39 & 42 & 44 & 53 & 65 & 58 & 68 & 69 \\
\hline Lithuania & 5 & 56 & 51 & 74 & 74 & 77 & 84 & 85 & 83 \\
\hline Luxembourg & 31 & 94 & 94 & 94 & 95 & 95 & 95 & 95 & 94 \\
\hline
\end{tabular}




\begin{tabular}{|l|r|r|r|r|r|r|r|r|r|r|r|} 
Hungary & 56 & 57 & 57 & 59 & 56 & 56 & 57 & 60 & 56 \\
\hline Malta & 53 & 36 & 39 & 38 & 32 & 38 & 43 & 41 & 38 \\
\hline Netherlands & 2 & 2 & 2 & 2 & 14 & 15 & 17 & 6 & 5 \\
\hline Austria & 19 & 21 & 20 & 19 & 20 & 20 & 27 & 23 & 19 \\
\hline Poland & $\mathbf{6 9}$ & $\mathbf{6 8}$ & $\mathbf{6 8}$ & $\mathbf{6 7}$ & $\mathbf{6 7}$ & $\mathbf{6 9}$ & $\mathbf{7 1}$ & $\mathbf{7 2}$ & $\mathbf{6 8}$ \\
\hline Portugal & 29 & 8 & 26 & 26 & 23 & 22 & 24 & 23 & 23 \\
\hline Romania & n.a. & n.a. & n.a. & n.a. & 57 & 84 & 82 & 79 & 76 \\
\hline Slovenia & 15 & 16 & 18 & 19 & 22 & 29 & 29 & 31 & 29 \\
\hline Slovakia & 80 & 81 & 94 & 94 & 93 & 78 & 83 & 93 & 96 \\
\hline Finland & 7 & 9 & 7 & 59 & 58 & 56 & 65 & 70 & 67 \\
\hline Sweden & 6 & 6 & 7 & 8 & 9 & 9 & 10 & 9 & 7 \\
\hline United Kingdom & 50 & 47 & 50 & 51 & 75 & 71 & 54 & 51 & 52 \\
\hline EU27* & $\mathbf{2 3}$ & $\mathbf{2 2}$ & $\mathbf{2 3}$ & $\mathbf{2 4}$ & $\mathbf{2 9}$ & $\mathbf{3 0}$ & $\mathbf{2 9}$ & $\mathbf{2 6}$ & $\mathbf{2 6}$ \\
\hline
\end{tabular}

Note: (n.a.) - not available

(*) For 2001-4 this refers to the EU15.

Source: European Central Bank, 'EU Banking Structures. September 2005' (European Central Bank 2005) 64;

European Central Bank, “'EU Banking Structures. September 2010’ (European Central Bank 2010) 46

Table 7 Number of credit institutions

\begin{tabular}{|c|c|c|c|c|c|c|c|c|c|}
\hline & 2001 & 2002 & 2003 & 2004 & 2005 & 2006 & 2007 & 2008 & 2009 \\
\hline Belgium & 112 & 111 & 108 & 104 & 100 & 105 & 110 & 105 & 104 \\
\hline Bulgaria & n.a. & n.a. & n.a. & n.a. & 34 & 32 & 29 & 30 & 30 \\
\hline Czech Republic & 119 & 84 & 77 & 70 & 56 & 57 & 56 & 54 & 56 \\
\hline Denmark & 203 & 178 & 203 & 202 & 197 & 191 & 189 & 171 & 164 \\
\hline Germany & 2526 & 2363 & 2225 & 2148 & 2089 & 2050 & 2026 & 1989 & 1948 \\
\hline Estonia & 7 & 7 & 7 & 9 & 11 & 14 & 15 & 17 & 18 \\
\hline Ireland** & 88 & 85 & 80 & 80 & 78 & 78 & 81 & 82 & 498 \\
\hline Greece & 61 & 61 & 59 & 62 & 62 & 62 & 63 & 66 & 66 \\
\hline Spain & 366 & 359 & 348 & 346 & 348 & 352 & 357 & 362 & 352 \\
\hline France & 1050 & 989 & 939 & 897 & 854 & 829 & 808 & 728 & 712 \\
\hline Italy & 843 & 821 & 801 & 787 & 792 & 807 & 821 & 818 & 801 \\
\hline Cyprus & 406 & 408 & 408 & 403 & 391 & 336 & 215 & 163 & 155 \\
\hline Latvia & 39 & 23 & 23 & 23 & 25 & 28 & 31 & 34 & 37 \\
\hline Lithuania & 54 & 68 & 71 & 74 & 78 & 78 & 80 & 84 & 84 \\
\hline Luxembourg & 189 & 177 & 169 & 162 & 155 & 156 & 156 & 152 & 147 \\
\hline Hungary & 240 & 227 & 222 & 221 & 214 & 212 & 206 & 197 & 190 \\
\hline Malta & 17 & 14 & 16 & 16 & 19 & 18 & 22 & 23 & 24 \\
\hline Netherlands & 561 & 539 & 481 & 461 & 401 & 345 & 341 & 302 & 295 \\
\hline Austria & 836 & 823 & 896 & 883 & 818 & 809 & 803 & 803 & 790 \\
\hline Poland* & 758 & 666 & 660 & 658 & 730 & 723 & 718 & 712 & 710 \\
\hline Portugal & 212 & 202 & 200 & 197 & 186 & 178 & 175 & 175 & 166 \\
\hline Romania & n.a. & n.a. & n.a. & n.a. & 40 & 39 & 42 & 43 & 42 \\
\hline Slovenia & 69 & 50 & 33 & 24 & 25 & 25 & 27 & 24 & 25 \\
\hline Slovakia & 21 & 20 & 21 & 21 & 23 & 24 & 26 & 26 & 26 \\
\hline Finland & 369 & 369 & 366 & 363 & 363 & 361 & 360 & 357 & 349 \\
\hline Sweden & 149 & 216 & 222 & 212 & 200 & 204 & 201 & 182 & 180 \\
\hline United Kingdom & 452 & 451 & 426 & 413 & 394 & 394 & 396 & 391 & 389 \\
\hline EU27*** & 9747 & 9311 & 9061 & 8836 & 8683 & 8507 & 8354 & 8090 & 8358 \\
\hline
\end{tabular}

Note: (n.a.) - not available

(*) For Poland, the data on the number of credit institutions includes credit unions.

(**) The jump in the number of credit institutions and local units in Ireland in 2009 is attributable to a reclassification of 419 credit unions as credit institutions.

(***) For 2001-4 this refers to the EU15. 
Source: European Central Bank, 'EU Banking Structures. September 2005’ (European Central Bank 2005) 52; European Central Bank, 'EU Banking Structures. September 2010’ (European Central Bank 2010) 34

\section{Table 8 Number of local units (branches)}

\begin{tabular}{|c|c|c|c|c|c|c|c|c|c|}
\hline & 2001 & 2002 & 2003 & 2004 & 2005 & 2006 & 2007 & 2008 & 2009 \\
\hline Belgium & 6168 & 555 & 4989 & 4837 & 4564 & 4574 & 4425 & 4316 & n.a. \\
\hline Bulgaria & n.a. & n.a. & n.a. & n.a. & 5629 & 5569 & 5827 & 6080 & 6038 \\
\hline Czech Republic & 1751 & 1722 & 1670 & 1785 & 1825 & 1877 & 1862 & 1993 & 1998 \\
\hline Denmark & 2376 & 2128 & 2118 & 2119 & 2122 & 2152 & 2194 & 2192 & 1996 \\
\hline Germany & 53931 & 50868 & 47244 & 45331 & 44044 & 40282 & 39777 & 39531 & 39411 \\
\hline Estonia & 210 & 198 & 197 & 203 & 230 & 245 & 266 & 257 & 213 \\
\hline Ireland** & 970 & 926 & 924 & 909 & 910 & 935 & 1158 & 895 & 1228 \\
\hline Greece & 3134 & 3263 & 3300 & 3403 & 3543 & 3699 & 385 & 4098 & 4078 \\
\hline Spain & 39012 & 39009 & 39750 & 40603 & 41979 & 43691 & 45500 & 46065 & 44431 \\
\hline France & 26049 & 26162 & 25789 & 26370 & 27075 & 40013 & 39560 & 39634 & 38479 \\
\hline Italy & 29267 & 29948 & 30501 & 30950 & 31504 & 32334 & 33230 & 34168 & 34035 \\
\hline Cyprus & 1009 & 993 & 983 & 977 & 951 & 941 & 921 & 923 & 930 \\
\hline Latvia & 590 & 567 & 581 & 583 & 586 & 610 & 682 & 658 & 624 \\
\hline Lithuania & 156 & 119 & 723 & 758 & 822 & 892 & 970 & 973 & 972 \\
\hline Luxembourg & 274 & 271 & 269 & 253 & 246 & 234 & 229 & 229 & b.d \\
\hline Hungary & 2950 & 2992 & 3003 & 2987 & 3125 & 3243 & 3387 & 3515 & 3551 \\
\hline Malta & 102 & 99 & 104 & 99 & 109 & 110 & 104 & 111 & 116 \\
\hline Netherlands & 4720 & 4269 & 3883 & 3798 & 3748 & 3456 & 3604 & 3421 & 3137 \\
\hline Austria & 4561 & 4466 & 4395 & 4360 & 4300 & 4258 & 4266 & 4243 & 4167 \\
\hline Poland* & 4080 & 4302 & 4394 & 5003 & 10074 & 10934 & 11607 & 12914 & 13292 \\
\hline Portugal & 5534 & 5348 & 5397 & 5371 & 5422 & 5618 & 6055 & 6417 & 6430 \\
\hline Romania & n.a. & n.a. & n.a. & n.a. & 3533 & 4470 & 6340 & 7375 & 6425 \\
\hline Slovenia & 717 & 721 & 720 & 706 & 693 & 696 & 711 & 698 & 706 \\
\hline Slovakia & 1052 & 102 & 1057 & 1113 & 1142 & 1175 & 1169 & 1258 & 1230 \\
\hline Finland & 1571 & 1572 & 1564 & 1585 & 1616 & 1756 & 1693 & 1672 & 1538 \\
\hline Sweden & 1986 & 1904 & 1906 & 1874 & 2003 & 2004 & 1988 & 2025 & 2147 \\
\hline United Kingdom & 14554 & 14392 & 14186 & 13902 & 13130 & 12880 & 12425 & 12360 & 12360 \\
\hline EU27*** & 206724 & 202809 & 199647 & 199879 & 214925 & 228648 & 233800 & 238021 & 229532 \\
\hline
\end{tabular}

Note: (n.a.) - not available

(*) For Poland, the data on the number of credit institutions includes credit unions.

(**) The jump in the number of credit institutions and local units in Ireland in 2009 is attributable to a reclassification of 419 credit unions as credit institutions.

$(* * *)$ For 2001-4 this refers to the EU15.

Source: European Central Bank, 'EU Banking Structures. September 2005’ (European Central Bank 2005) 52;

European Central Bank, 'EU Banking Structures. September 2010' (European Central Bank 2010) 34

Table 9 Number of residents per credit institution local unit

\begin{tabular}{|c|c|c|c|c|c|c|c|c|c|}
\hline & 2001 & 2002 & 2003 & 2004 & 2005 & 2006 & 2007 & 2008 & 2009 \\
\hline Belgium & 1667 & 18613 & 2079 & 2154 & 2295 & 2305 & 2400 & 2481 & n.a. \\
\hline Bulgaria & n.a. & n.a. & n.a. & n.a. & 1371 & 1379 & 1311 & 1251 & 1253 \\
\hline Czech Republic & 5839 & 5924 & 6109 & 5718 & 5608 & 5470 & 5544 & 5233 & 5251 \\
\hline Denmark & 2255 & 2526 & 2545 & 2550 & 2554 & 2526 & 2489 & 2505 & 2767 \\
\hline Germany & 1527 & 1621 & 1747 & 1820 & 1872 & 2045 & 2068 & 2077 & 2077 \\
\hline Estonia & 6510 & 6874 & 6883 & 6680 & 5861 & 5490 & 5045 & 5218 & 6291 \\
\hline Ireland** & 3978 & 4240 & 4319 & 4465 & 4559 & 4549 & 3763 & 4964 & 3634 \\
\hline Greece & 3494 & 3367 & 3341 & 3249 & 3134 & 3014 & 29073 & 2742 & 2761 \\
\hline Spain & 1044 & 1059 & 1057 & 1051 & 1034 & 1009 & 986 & 990 & 1034 \\
\hline
\end{tabular}




\begin{tabular}{|l|r|r|r|r|r|r|r|r|r|}
\hline France & 2346 & 2352 & 2401 & 2363 & 2325 & 1584 & 1612 & 1618 & 1676 \\
\hline Italy & 1947 & 1909 & 1889 & 1880 & 1860 & 1823 & 1787 & 1751 & 1771 \\
\hline Cyprus & 696 & 715 & 736 & 757 & 797 & 821 & 851 & 859 & 858 \\
\hline Latvia & 3992 & 4125 & 4002 & 3967 & 3927 & 3751 & 3337 & 3444 & 3614 \\
\hline Lithuania & 22314 & 29151 & 4777 & 4533 & 4153 & 3805 & 3480 & 3451 & 3435 \\
\hline Luxembourg & 1613 & 1646 & 1673 & 1791 & 1890 & 2021 & 2096 & 2135 & n.a. \\
\hline Hungary & 3453 & 3395 & 3373 & 3384 & 3228 & 3105 & 2969 & 2856 & 2822 \\
\hline Malta & 3853 & 4000 & 3827 & 4051 & 3697 & 3691 & 3933 & 3712 & 3560 \\
\hline Netherlands & 3399 & 3782 & 4178 & 4285 & 4354 & 4728 & 4544 & 4806 & 5268 \\
\hline Austria & 1763 & 1810 & 1847 & 1875 & 1913 & 1942 & 1946 & 1965 & 2007 \\
\hline Poland* & $\mathbf{9 3 7 5}$ & $\mathbf{8 ~ 8 8 7}$ & $\mathbf{8 6 9 3}$ & $\mathbf{7 6 3 1}$ & $\mathbf{3 7 8 8}$ & $\mathbf{3 4 8 7}$ & $\mathbf{3 2 8 4}$ & $\mathbf{2 9 5 2}$ & $\mathbf{2 8 6 7}$ \\
\hline Portugal & 1860 & 1939 & 1935 & 1955 & 1946 & 1884 & 1752 & 1655 & 1653 \\
\hline Romania & n.a. & n.a. & n.a. & n.a. & 6121 & 4829 & 3397 & 2916 & 3340 \\
\hline Slovenia & 2778 & 2767 & 2772 & 2829 & 2887 & 2885 & 2840 & 2897 & 2892 \\
\hline Slovakia & 5136 & 52735 & 5090 & 4836 & 4717 & 4588 & 4617 & 4297 & 4405 \\
\hline Finland & 3302 & 3309 & 3333 & 3298 & 3246 & 2999 & 3124 & 3178 & 3471 \\
\hline Sweden & 4479 & 4688 & 4700 & 4799 & 4508 & 4531 & 4602 & 4571 & 4351 \\
\hline United Kingdom & 4057 & 4122 & 4198 & 4304 & 4588 & 4704 & 4907 & 4966 & 4997 \\
\hline EU27*** & $\mathbf{2 1 9 6}$ & $\mathbf{2 2 4 8}$ & $\mathbf{2 2 9 4}$ & $\mathbf{2 3 0 2}$ & $\mathbf{2 2 9 9}$ & $\mathbf{2 ~ 1 6 1}$ & $\mathbf{2 ~ 1 2 3}$ & $\mathbf{2 ~ 0 9 5}$ & $\mathbf{2 1 8 0}$ \\
\hline Note: (na) & & & & & & & &
\end{tabular}

Note: (n.a.) - not available

(*) For Poland, the data on the number of credit institutions includes credit unions.

(**) The jump in the number of credit institutions and local units in Ireland in 2009 is attributable to a reclassification of 419 credit unions as credit institutions.

(***) For 2001-4 this refers to the EU15.

Source: European Central Bank, 'EU Banking Structures. September 2005' (European Central Bank 2005) 65;

European Central Bank, 'EU Banking Structures. September 2010' (European Central Bank 2010) 47

Table 10 Number of credit institution employees

\begin{tabular}{|c|c|c|c|c|c|c|c|c|c|}
\hline & 2001 & 2002 & 2003 & 2004 & 2005 & 2006 & 2007 & 2008 & 2009 \\
\hline Belgium & 76104 & 75370 & 73553 & 71347 & 69481 & 67957 & 67080 & 65985 & n.a. \\
\hline Bulgaria & n.a. & n.a. & n.a. & n.a. & 23636 & 25633 & 30953 & 33258 & 34290 \\
\hline Czech Republic & 42999 & 40534 & 39658 & 38666 & 37943 & 37825 & 40037 & 39882 & 38394 \\
\hline Denmark & 48538 & 47613 & 46443 & 46372 & 47579 & 46394 & 49644 & 52830 & 50101 \\
\hline Germany & 772100 & 753950 & 725550 & 712300 & 705000 & 692500 & 691300 & 685550 & n.a. \\
\hline Estonia & 3949 & 3934 & 4280 & 4455 & 5029 & 5681 & 6319 & 6144 & 5693 \\
\hline Ireland** & 40928 & 36585 & 35658 & 35564 & 37702 & 39154 & 41865 & 40507 & 38178 \\
\hline Greece & 59624 & 60495 & 61074 & 59337 & 61295 & 62171 & 64713 & 66163 & 65673 \\
\hline Spain & 244781 & 243429 & 243462 & 246006 & 252831 & 261890 & 275506 & 276497 & 267383 \\
\hline France & 424615 & 428438 & 425041 & 429347 & 442230 & 484557 & 497384 & 492367 & n.a. \\
\hline Italy & 341299 & 340440 & 336661 & 336354 & 335726 & 339091 & 340443 & 337962 & 322575 \\
\hline Cyprus & 10115 & 10613 & 10480 & 10617 & 10799 & 10845 & 11286 & 12554 & 12513 \\
\hline \begin{tabular}{|l|} 
Latvia \\
\end{tabular} & 8172 & 8267 & 8903 & 9655 & 10477 & 11656 & 12826 & 13905 & 12365 \\
\hline \begin{tabular}{|l} 
Lithuania \\
\end{tabular} & 8796 & 8420 & 7557 & 7266 & 7637 & 8624 & 10303 & 11080 & 10902 \\
\hline Luxembourg & 23894 & 23300 & 22513 & 22549 & 23224 & 24752 & 26128 & 27208 & 26416 \\
\hline Hungary & 34054 & 35045 & 35725 & 35558 & 37527 & 39302 & 41905 & 43620 & 42607 \\
\hline Malta & 3584 & 3459 & 3401 & 3353 & 3383 & 3515 & 3756 & 3906 & 3834 \\
\hline Netherlands & 131230 & 125911 & 120539 & 118032 & 120165 & 116500 & 114424 & 116000 & 110000 \\
\hline Austria & 74606 & 74048 & 73308 & 72858 & 75303 & 76323 & 77731 & 78754 & 77246 \\
\hline Poland & 168529 & 161814 & 154569 & 150037 & 158130 & 162125 & 173955 & 188969 & 183064 \\
\hline \begin{tabular}{|l|} 
Portugal* \\
\end{tabular} & 55538 & 55679 & 54350 & 53230 & 54035 & 58213 & 60979 & 62377 & 62221 \\
\hline Romania & n.a. & n.a. & n.a. & n.a. & 52452 & 58536 & 66039 & 71622 & 67898 \\
\hline Slovenia & 11578 & 11855 & 11816 & 11602 & 11726 & 11838 & 12051 & 12284 & 12188 \\
\hline Slovakia & 20118 & 18452 & 18350 & 18261 & 19850 & 19525 & 19779 & 20598 & 18750 \\
\hline Finland & 26733 & 27190 & 26667 & 25377 & 23644 & 24769 & 25025 & 25699 & 24879 \\
\hline
\end{tabular}




\begin{tabular}{|l|r|r|r|r|r|r|r|r|r|} 
Sweden & 42001 & 42357 & 40169 & 39181 & 44943 & 47069 & 48457 & 50115 & 49071 \\
\hline United Kingdom & 506278 & 501787 & 487772 & 484535 & 534482 & 521476 & 505661 & 495493 & 471095 \\
\hline EU27*** & $\mathbf{3 1 8 0 ~ 1 6 3}$ & $\mathbf{3 1 3 8 ~ 9 8 5}$ & $\mathbf{3 0 6 7 ~ 4 9 9}$ & $\mathbf{3 0 4 1 ~ 8 5 9}$ & $\mathbf{3 2 0 6} \mathbf{2 2 9}$ & $\mathbf{3 2 5 7 9 2 1}$ & $\mathbf{3 3 1 5} \mathbf{5 4 9}$ & $\mathbf{3 3 3 1 ~ 3 2 9}$ & $\mathbf{2 ~ 0 0 7 ~ 3 3 6}$ \\
\hline
\end{tabular}

Note: (n.a.) - not available

(*) For Portugal the increase in the number of employees in 2006 was mainly due to the incorporation of backoffice operations (and staff) previously organized through jointly controlled entities into two of the main Portuguese banks.

(**) The number of employees in Ireland excludes employees in credit unions (only applicable from 2009 on). (***) For 2001-4 this refers to the EU15.

Source: European Central Bank, 'EU Banking Structures. September 2005' (European Central Bank 2005) 53; European Central Bank, 'EU Banking Structures. September 2010' (European Central Bank 2010) 35

Table 11 Assets of credit institutions per employee (EUR '000s)

\begin{tabular}{|c|c|c|c|c|c|c|c|c|c|}
\hline & 2001 & 2002 & 2003 & 2004 & 2005 & 2006 & 2007 & 2008 & 2009 \\
\hline Belgium & 10199 & 10274 & 11265 & 12816 & 15188 & 16509 & 19347 & 19258 & n.a. \\
\hline Bulgaria & n.a. & n.a. & n.a. & n.a. & 738 & 870 & 1009 & 1107 & 1107 \\
\hline Czech Republic & 1818 & 1955 & 1967 & 2238 & 2659 & 3037 & 3501 & 3887 & 4173 \\
\hline Denmark & 9360 & 10642 & 11766 & 13092 & 15692 & 17694 & 19706 & 20666 & 22046 \\
\hline Germany & 8119 & 8449 & 8812 & 9244 & 9683 & 10283 & 10939 & 11488 & n.a. \\
\hline Estonia & 1107 & 1327 & 1475 & 1916 & 2362 & 2707 & 3260 & 3591 & 3748 \\
\hline Ireland** & 10313 & 12973 & 16130 & 20317 & 24983 & 30090 & 31944 & 34863 & 34669 \\
\hline Greece & 3400 & 3333 & 3490 & 3884 & 4585 & 5068 & 5923 & 6982 & 7463 \\
\hline Spain & 5098 & 5515 & 6173 & 6981 & 8515 & 9648 & 10908 & 12229 & 12840 \\
\hline France & 8876 & 8943 & 9397 & 10284 & 11472 & 11821 & 13435 & 14674 & n.a. \\
\hline Italy & 5426 & 5946 & 6313 & 6766 & 7475 & 8237 & 9787 & 10754 & 11445 \\
\hline Cyprus & 4179 & 3858 & 3997 & 4384 & 5792 & 7065 & 8231 & 9411 & 11138 \\
\hline Latvia & 891 & 877 & 953 & 1157 & 1501 & 1947 & 2403 & 2319 & 2420 \\
\hline Lithuania & 496 & 595 & 850 & 1171 & 1723 & 2011 & 2312 & 2395 & 2401 \\
\hline Luxembourg & 30175 & 28438 & 29137 & 30826 & 34121 & 33919 & 35037 & 34239 & 30189 \\
\hline Hungary & 1129 & 1243 & 1533 & 1827 & 2086 & 2384 & 2589 & 2858 & 2961 \\
\hline Malta & 4398 & 4705 & 5235 & 6140 & 8039 & 8545 & 10066 & 10825 & 10757 \\
\hline Netherlands & 9646 & 10773 & 12228 & 14213 & 14129 & 15821 & 18950 & 19237 & 20155 \\
\hline Austria & 7685 & 7489 & 8000 & 8720 & 9577 & 10348 & 11459 & 13564 & 13419 \\
\hline Poland & 792 & 717 & 671 & 879 & 1033 & 1170 & 1345 & 1390 & 1498 \\
\hline Portugal* & 5373 & 5574 & 6416 & 6488 & 6666 & 6822 & 7207 & 7729 & 8360 \\
\hline Romania & n.a. & n.a. & n.a. & n.a. & 675 & 887 & 1092 & 1180 & 1272 \\
\hline Slovenia & 1536 & 1687 & 1823 & 2108 & 2570 & 2943 & 3609 & 3990 & 4382 \\
\hline Slovakia & 1066 & 1287 & 1294 & 1590 & 1906 & 2517 & 2935 & 3180 & 2905 \\
\hline Finland & 6113 & 6093 & 6969 & 8371 & 9919 & 10297 & 11497 & 14939 & 15581 \\
\hline Sweden & 10769 & 11210 & 12609 & 14878 & 14669 & 16613 & 17643 & 18109 & 19045 \\
\hline United Kingdom & 11515 & 11666 & 12659 & 14302 & 11034 & 13540 & 19963 & 17841 & 19998 \\
\hline EU27*** & 7754 & 8048 & 8631 & 9526 & 9526 & 10526 & 12403 & 12673 & 20995 \\
\hline
\end{tabular}

Note: (n.a.) - not available

(*) For Portugal the increase in the number of employees in 2006 was mainly due to the incorporation of backoffice operations (and staff) previously organized through jointly controlled entities into two of the main Portuguese banks.

(**) The number of employees in Ireland excludes employees in credit unions (only applicable from 2009 on). (***) For 2001-4 this refers to the EU15.

Source: European Central Bank, 'EU Banking Structures. September 2005' (European Central Bank 2005) 53; European Central Bank, 'EU Banking Structures. September 2010' (European Central Bank 2010) 35 
Table 12 Financial assets of households in Poland (PLN bn)

\begin{tabular}{|r|c|c|c|c|c|c|c|c|c|c|}
\hline & $\mathbf{2 0 0 0}$ & $\mathbf{2 0 0 1}$ & $\mathbf{2 0 0 2}$ & $\mathbf{2 0 0 3}$ & $\mathbf{2 0 0 4}$ & $\mathbf{2 0 0 5}$ & $\mathbf{2 0 0 6}$ & $\mathbf{2 0 0 7}$ & $\mathbf{2 0 0 8}$ & $\mathbf{2 0 0 9}$ \\
\hline Assets in Open Pension Funds included & 2.3 & 19.4 & 31.6 & 44.8 & 62.6 & 86.1 & 116.6 & 140 & 138.3 & 178.6 \\
\hline
\end{tabular}
Source: Jacek Osiński, Paweł Sobolewski, Dobiesław Tymoczko (eds), Rozwój systemu finansowego w Polsce $w$ 2006r. (NBP 2008) 21; Paweł Sobolewski, Dobiesław Tymoczko (eds), Rozwój systemu finansowego w Polsce w 2009r. (NBP 2010) 16

Table 13 Financial assets of Polish households as a percentage of GDP

\begin{tabular}{|c|c|c|c|c|c|c|c|c|c|c|}
\hline & 2000 & 2001 & 2002 & 2003 & 2004 & 2005 & 2006 & 2007 & 2008 & 2009 \\
\hline Assets as & 37.7 & 43.1 & 45.2 & 47.5 & 47 & \begin{tabular}{|c|}
51.8 \\
\end{tabular} & 60 & 63.8 & 58 & \\
\hline
\end{tabular}

Source: Jacek Osiński, Paweł Sobolewski, Dobiesław Tymoczko (eds.), Rozwój systemu finansowego w Polsce w 2006r. (NBP 2008) 21; Paweł Sobolewski, Dobiesław Tymoczko (eds.), Rozwój systemu finansowego w

Polsce w 2009r. (NBP 2010) 16

Table 14 Structure of the financial assets of Polish households (in \%)

\begin{tabular}{|c|c|c|c|c|c|c|c|c|c|}
\hline & 2001 & 2002 & 2003 & 2004 & 2005 & 2006 & 2007 & 2008 & 2009 \\
\hline Bank deposits & 65.4 & 58.2 & 52.4 & 47.7 & 42.9 & 37.2 & 34.7 & 44.3 & 43.8 \\
\hline Funds on accounts with open pension funds & 5.8 & 8.6 & 11.2 & 14.4 & 16.9 & 18.3 & 18.6 & 18.7 & 20.8 \\
\hline Participation units of investment funds & 3.0 & 5.6 & 7.6 & 7.9 & 11.4 & 14.8 & 17.1 & 8.3 & 9.1 \\
\hline Unit-linked assets and life insurance saving premiums & 6.2 & 7.0 & 7.5 & 8.1 & 8.2 & 8.3 & 8.4 & 9.2 & 7.9 \\
\hline Stock listed on the Warsaw Stock Exchange & 2.9 & 2.2 & 2.8 & 4.3 & 5.1 & 7.2 & 8.1 & 3.8 & 4.9 \\
\hline Treasury securities & 4.8 & 5.6 & 4.8 & 4.7 & 3.3 & 2.0 & 1.4 & 1.8 & 1.5 \\
\hline Deposits with credit unions & 0.5 & 0.6 & 0.8 & 0.9 & 1.0 & 0.9 & 0.9 & 1.2 & 1.3 \\
\hline Cash in circulation (excluding bank vault cash) & 11.4 & 11.5 & 12.3 & 11.7 & 11.2 & 10.8 & 10.3 & 12.3 & 10.4 \\
\hline
\end{tabular}

Source: Jacek Osiński, Paweł Sobolewski, Dobiesław Tymoczko (eds.), Rozwój systemu finansowego w Polsce w 2006r. (NBP 2008) 24; Paweł Sobolewski, Dobiesław Tymoczko (eds), Rozwój systemu finansowego w Polsce w 2009r. (NBP 2010) 16

Table 15 GDP per capita in Purchasing Power Standards (EU27=100)

\begin{tabular}{|c|c|c|c|c|c|c|c|c|c|}
\hline & 2001 & 2002 & 2003 & 2004 & 2005 & 2006 & 2007 & 2008 & 2009 \\
\hline Belgium & 124 & 125 & 123 & 121 & $120^{\mathrm{b}}$ & 118 & 116 & 115 & 116 \\
\hline Bulgaria & 30 & 32 & 34 & 35 & $37^{\mathrm{b}}$ & 38 & 40 & 44 & 44 \\
\hline Czech Republic & 70 & 70 & 73 & 75 & $76^{\mathrm{b}}$ & 77 & 80 & 80 & 82 \\
\hline \begin{tabular}{|l} 
Denmark \\
\end{tabular} & 128 & 128 & 124 & 126 & $125^{b}$ & 124 & 123 & 123 & 121 \\
\hline Germany & 117 & 115 & 116 & 116 & $117^{b}$ & 116 & 116 & 116 & 116 \\
\hline Estonia & 46 & 50 & 54 & 57 & $62^{\mathrm{b}}$ & 66 & 69 & 68 & 64 \\
\hline Ireland & 132 & 138 & 141 & 142 & $144^{\mathrm{b}}$ & 145 & 147 & 133 & 127 \\
\hline Greece & 86 & 90 & 93 & $94^{\mathrm{p}}$ & $91^{\mathrm{b}}$ & $93^{\mathrm{p}}$ & $92^{\mathrm{p}}$ & $94^{\mathrm{p}}$ & $94^{\mathrm{r}}$ \\
\hline Spain & 98 & 100 & 101 & 101 & $102^{b}$ & 104 & 105 & 103 & 103 \\
\hline France & 115 & 115 & 111 & 110 & $110^{\mathrm{b}}$ & 108 & 108 & 106 & 107 \\
\hline Italy & 118 & 112 & 111 & 107 & $105^{b}$ & 104 & 104 & 104 & 104 \\
\hline Cyprus & 91 & 89 & 89 & 90 & $91^{\mathrm{b}}$ & 91 & 93 & 97 & 98 \\
\hline Latvia & 39 & 41 & 43 & 46 & $49^{b}$ & 52 & 56 & 56 & 52 \\
\hline Lithuania & 41 & 44 & 49 & 50 & $53^{\mathrm{b}}$ & 55 & 59 & 61 & 55 \\
\hline Luxembourg & 234 & 240 & 247 & 253 & $254^{\mathrm{b}}$ & 270 & 275 & 279 & 271 \\
\hline Hungary & 59 & 62 & 63 & 63 & $63^{\mathrm{b}}$ & 63 & 62 & 64 & 65 \\
\hline Malta & 78 & 79 & 78 & 77 & $78^{b}$ & 76 & 76 & 79 & 81 \\
\hline Netherlands & 134 & 133 & 129 & 129 & $131^{b}$ & 131 & 132 & 134 & 131 \\
\hline Austria & 125 & 126 & 127 & 127 & $124^{\mathrm{b}}$ & 125 & 123 & 124 & 124 \\
\hline
\end{tabular}




\begin{tabular}{|c|c|c|c|c|c|c|c|c|c|}
\hline Poland & 48 & 48 & 49 & 51 & $51^{\mathrm{b}}$ & 52 & 54 & 56 & 61 \\
\hline Portugal & 80 & 80 & 79 & 77 & $78^{b}$ & 79 & 79 & 78 & $80^{\mathrm{p}}$ \\
\hline Romania & 28 & 29 & 31 & 34 & $35^{b}$ & 38 & 42 & 47 & 46 \\
\hline Slovenia & 80 & 82 & 83 & 86 & $87^{\mathrm{b}}$ & 88 & 88 & $91^{\mathrm{b}}$ & $88^{\mathrm{b}}$ \\
\hline Slovakia & 52 & 54 & 55 & 57 & $60^{\mathrm{b}}$ & 63 & 68 & 72 & 73 \\
\hline Finland & 115 & 115 & 112 & 116 & $114^{b}$ & 114 & 117 & 118 & 113 \\
\hline Sweden & 122 & 122 & 124 & 126 & $122^{b}$ & 123 & 125 & 123 & 119 \\
\hline United Kingdom & 120 & 120 & 122 & 124 & $122^{\mathrm{b}}$ & 120 & 116 & 115 & 112 \\
\hline EU27 & 100 & 100 & 100 & 100 & $100^{b}$ & 100 & 100 & 100 & 100 \\
\hline
\end{tabular}

Note: (b) - break in series;

(p) - provisional value

Source: Eurostat 